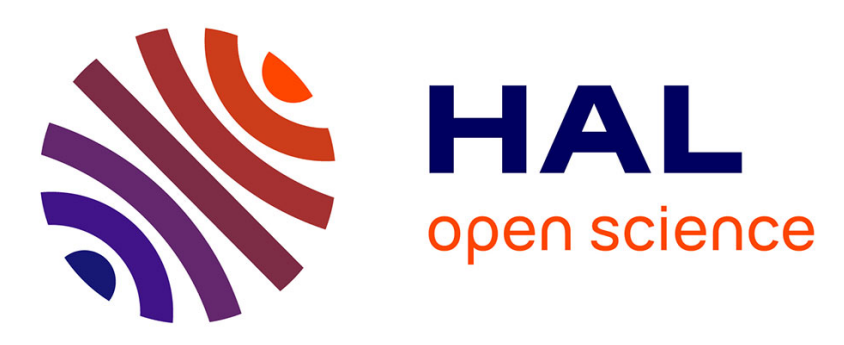

\title{
Imagerie combinée géoélectriqueradar géologique des cavités souterraines de la ville de Zaouit Ech Cheikh (Maroc)
}

Kamal El Khammari, Abdessamad Najine, Mohammed Jaffal, Tahar Aifa, Mahjoub Himi, Diego Vasquez, Albert Casas, Pierre Andrieux

\section{To cite this version:}

Kamal El Khammari, Abdessamad Najine, Mohammed Jaffal, Tahar Aifa, Mahjoub Himi, et al.. Imagerie combinée géoélectriqueradar géologique des cavités souterraines de la ville de Zaouit Ech Cheikh (Maroc). Comptes Rendus Géoscience, 2007, 339 (7), pp.460-467. 10.1016/j.crte.2007.06.001 . insu-00179716

\section{HAL Id: insu-00179716 https://hal-insu.archives-ouvertes.fr/insu-00179716}

Submitted on 16 Oct 2007

HAL is a multi-disciplinary open access archive for the deposit and dissemination of scientific research documents, whether they are published or not. The documents may come from teaching and research institutions in France or abroad, or from public or private research centers.
L'archive ouverte pluridisciplinaire HAL, est destinée au dépôt et à la diffusion de documents scientifiques de niveau recherche, publiés ou non, émanant des établissements d'enseignement et de recherche français ou étrangers, des laboratoires publics ou privés. 


\title{
Imagerie combinée géoélectrique- radar géologique des cavités souterraines de la ville de Zaouit Ech Cheikh (Maroc)
}

\author{
Kamal El Khammari ${ }^{\mathbf{a}}$, Abdessamad Najine ${ }^{\mathbf{b}}$, Mohammed Jaffal ${ }^{\text {c, Tahar Aïfa }}{ }^{\text {d,* }}$, \\ Mahjoub Himi ${ }^{\mathbf{e}}$, Diego Vásquez ${ }^{\mathbf{e}}$, Albert Casas ${ }^{\mathbf{e}}$, Pierre Andrieux ${ }^{\mathbf{f}}$ \\ a Université de Mohammed I, Faculté des Sciences, Laboratoire de géophysique, géodynamique et géoressources \\ (L3G), B.P. 524, Oujda, Maroc \\ b Université Cadi Ayyad, Faculté des Sciences et Techniques, BP 523, Béni-Mellal, Maroc \\ c Université Cadi Ayyad, Faculté des Sciences et Techniques, BP 549, Marrakech, Maroc \\ d Géosciences-Rennes, CNRS UMR6118, Université de Rennes 1, Bat.15, Campus de Beaulieu, 35042 Rennes \\ Cedex, France \\ e Universidad de Barcelona, Department de Geoquímica, Petrologia i Prospecció, Facultat de Geologia, Spain \\ f Université Pierre et Marie-Curie (Paris 6), UMR7619 Sisyphe, Case 105, 4 Place Jussieu, 75252 Paris Cedex \\ 05, France
}

*correspondance, e-mail: tahar.aifa@univ-rennes1.fr

\section{Résumé}

Le sous-sol de la ville de Zaouit Ech Cheikh abrite un réseau dense de cavités et grottes souterraines. Ces vides de sub-surface sont à l'origine de nombreux désordres et instabilités des constructions et des infrastructures. Afin de tester l'efficacité des méthodes géophysiques à détecter ces cavités, les techniques de tomographie électrique et du radar géologique ont été mises en œuvre le long des principales artères de la ville. Les résultats obtenus révèlent l'existence de cavités souterraines dans l'ensemble de la superficie couverte. Ils montrent également l'utilité de ces méthodes géophysiques combinées comme outils de cartographie des vides souterrains en milieu urbain.

Mots clés : cavité souterraine, tomographie électrique, radar géologique, Zaouit Ech Cheikh. 


\title{
Combined geoelectrical-GPR mapping of underground cavities in Zaouit Ech Cheikh city (Morocco)
}

\begin{abstract}
The basement of the Zaouit Ech Cheik city shelters a dense network of underground cavities. These sub-surface voids make many buildings or infrastructures unstable or cause them to collapse. To test the efficiency of geophysical methods in detecting cavities, 2D electrical tomography and ground penetrating radar (GPR) studies were carried out along of the main streets of the city. The results obtained reveal the existence of a large number of underground cavities throughout the investigated area. They also show the interest of such combined geophysical methods as tools for mapping underground holes in an urban area.
\end{abstract}

Keywords: underground cavities, electrical tomography, GPR, Zaouit Ech Cheikh

\section{Abridged version}

The city of Zaouit Ech Cheikh (Morocco) is located in the hilly zone which delineates the High Atlas Mountain (Fig. 1). Its basement is mainly composed of Maestrichtien-Eocene sedimentary formations [3]. The sedimentary series is made of limestones [6] the dissolving of which led to the formation of a complex network of underground karsts. In this area, artificial cavities formerly dug by inhabitants to satisfy their domestic needs were also found. The existence of such underground voids makes buildings and infrastructures very unstable (Photo 1). So it is necessary to locate and characterize such cavities (geometry, depth) in order to guide the town planning scheme within the urban perimeter of the city and to find solutions to avoid collapses. 
The purpose of the present paper is to carry out combined 2D electrical tomography and ground penetrating radar (GPR) studies to map the sub-surface and detect cavities. The use of such geophysical tools to recognize underground voids has already given satisfactory results $[1,2,4,7,8,12,15,19]$. Measurements were conducted along the main streets of the city following a path to get more sub-surface information (Fig. 2). During this survey, we noticed many signs of instability and some collapses in the neighbouring buildings. They may indicate the existence of voids beneath these buildings.

Electrical tomography was applied along two profiles (E1 and E2) (Figs. 2,3) using a multi-electrodes device [10] in a Wenner configuration [17,20]. Electrodes were $4 \mathrm{~m}$ apart and connected to a Syscal Junior resistivity-meter manufactured by Iris Instruments. Acquired data are presented as pseudosections (Fig. 3). Electrical resistivity models of the ground are calculated from the data using the inverse method of Loke and Barker (1996) [13].

The GPR measurements were performed along fourteen profiles (1 to 14) with a Ramac system of Mala Geosciences, using a $200 \mathrm{MHz}$ antenna (Figs. 2,3; Photo 2). In this method, a transmitting antenna generates an electromagnetic impulse which propagates in the ground and is reflected on the contacts between geological layers characterized by dielectric permittivity contrasts [18]. The reflected impulse is recorded by a receiving antenna [5, 9, 11].

The geophysical results recorded along the profiles E1 and E2 are presented in figure 3. We chose these profiles, carried out with two methods, because they allow a comparison between the two sets of geophysical data. Electrical tomography measurements are presented as pseudosections or panels that, through inversion, lead to establish ground resistivity models. They show several local resistive zones (Fig. 3a, f) that we numbered from one to ten, superimposed on GPR images (Fig. 3c, h).

The geophysical survey was complemented with a field investigation in order to gather information on the underground cavities of the prospected area. The investigation is based on 
collecting information from the inhabitants who had a relatively accurate knowledge of known cavities. It also includes a detailed mapping of the indices of instabilities or depressions visible on some buildings and roadway systems (Fig. 2). The results of this investigation for the profiles E1 and E2 are shown in figure 3e,j.

The agreement of the geophysical data and investigation information confirms the assumption of the existence of underground cavities. It is shown from the electrical resistivity anomalies 3, 4 and 5 and 9 and 10 evidenced respectively on E1 and E2 profiles that we interpret as cavities (Fig. 3d,i). However, the information provided by the investigation was taken only as an indication considering inaccuracies in the location of cavities. Thus, the resistive zones 2 on profile E1 and 5 on profile E2, which match with strong amplitude reflections, probably correspond to underground vacuums although they are not perfectly centred on the cavities underlined by the investigation. Moreover, taking into account that the results of the investigation could not highlight all of cavities, we interpret anomalies 1 and 2 of profile E1 and 6 of profile E2 as underground vacuums because they correspond to resistive zones which coincide with energetic reflections (Fig. 3c,h).

Furthermore, we must point out that the most important resistive zone which corresponds to anomaly 1 on profile E1, would not correspond to an underground cavity because it is superimposed on the GPR section with a perfectly horizontal layering which probably reflects the initial aspect of the basement of the foundations of the Zaouit Ech Cheikh city.

The underground cavities highlighted by the present study on profiles E1 and E2 are shown in figure 3e,j. These cavities show different shapes and sizes (Photo 3). Some of them are lengthened vertically; they could correspond to septic tanks (C1, C'3 and C'4). The other cavities would represent either karsts or zones from which sandy materials were removed for building. 


\section{Introduction}

Le sous-sol de la ville de Zaouit Ech Cheikh (Fig. 1) abrite un grand nombre de cavités souterraines naturelles de type karstique ou artificielles liées aux activités humaines. Ces cavités sont souvent à l'origine d'affaissements, de fontis, voire même d'effondrements dangereux tant pour les vies que pour les biens (Photo 1). Les investigations que nous avons menées sur le terrain permettent de se rendre compte de l'ampleur de ces vides souterrains et de leur impact sur les instabilités des constructions et des infrastructures de la ville de Zaouit Ech Cheikh. Ainsi, nous avons pu constater que les cavités ouvertes suite à des effondrements sont toutes remplies d'air. Elles peuvent atteindre plusieurs mètres cubes de volume et la profondeur de leur toit peut varier de 80 à $500 \mathrm{~cm}$.

Partant de ces considérations, le contrôle et l’orientation des aménagements au sein du périmètre urbain de la ville de Zaouit Ech Cheikh nécessitent la cartographie détaillée et la caractérisation précise de ces cavités. Les contrastes notables des propriétés physiques existant entre les cavités (air) et leur encaissant offrent un contexte très favorable pour les caractériser par méthodes géophysiques.

On se propose dans cette étude de mettre en œuvre les techniques de tomographie électrique et du radar géologique pour la détection et la délimitation des cavités souterraines en milieu urbain. Ces deux méthodes géophysiques ont déjà été utilisées avec succès pour la reconnaissance de cavités souterraines dans d'autres contextes [1, 2, 4, 7, 8, 12, 15, 19].

\section{Contexte géologique}

La ville de Zaouit Ech Cheikh est située au pied de la chaîne du Haut-Atlas dans la zone bordière des collines (Fig. 1). Dans cette zone, la série stratigraphique est essentiellement formée de calcaires et calcaires dolomitiques attribués au Lias inférieur reposant généralement sur des formations permo-triasiques composées d'argiles rouges et de 
basaltes doléritiques. Les affleurements d’âge quaternaire consistent en des dépôts travertineux tufeux, gréseux et même conglomératiques de puissance variable.

Dans ce contexte régional de nature essentiellement carbonatée, les phénomènes de dissolution ont entraîné l'apparition d'un système complexe de cavités karstiques de taille variable et de forme irrégulière. A ces vides souterrains d'origine naturelle, s’ajoutent les cavités artificielles anciennement creusées par les habitants pour l'extraction des faciès gréseux utilisés comme matériaux de construction. Ainsi, aujourd’hui, le sous-sol de la ville de Zaouit Ech Cheikh abrite un important réseau de cavités.

\section{Méthodologie}

Le programme de reconnaissance comprenait 14 profils de radar géologique et 2 profils de tomographie électrique, planifiés au préalable à l’intérieur du périmètre urbain de la ville (Fig. 2). L'objectif de nos mesures est de mettre en évidence les vides souterrains responsables des désordres et des indices d’instabilités que montrent de nombreuses constructions.

La tomographie électrique est une méthode de prospection géophysique qu’on utilise pour imager la résistivité électrique des terrains de sub-surface. Elle consiste en la mise en œuvre d'un dispositif multi-électrodes permettant d'acquérir un grand nombre de mesures correspondant aux différentes combinaisons de quatre électrodes [10]. Dans la présente étude la configuration Wenner a été adoptée. Le dispositif de mesures est constitué d’un résistivitémètre de type Syscal Junior de la société Iris Instruments, connecté à un agencement de 48 électrodes espacées de $4 \mathrm{~m}$. Les données recueillies sont présentées sous forme de panneaux électriques sur lesquels les mesures sont reportées au milieu du dispositif d’acquisition à une cote qui correspond à la valeur de la séparation courante des électrodes mobiles [17, 20]. Des modèles du sous-sol montrant la distribution en coupe de la résistivité, sont calculés à partir des panneaux en utilisant la méthode d’inversion des données de Loke et Barker (1996) [13]. 
La seconde méthode géophysique utilisée dans cette étude, est le radar géologique dont le principe est similaire à celui de la sismique réflexion, mais où les ondes acoustiques sont remplacées par des ondes électromagnétiques [5, 9, 11]. Les mesures ont été effectuées en utilisant un équipement de type Ramac manufacturé par de la société Mala Géosciences, avec des antennes dont la fréquence centrale est de $200 \mathrm{MHz}$ (Photo 2). La signature d'une cavité est un contraste d'amplitude plus ou moins prononcé sur le radargramme, proportionnel au rapport des permittivités effectives de l'encaissant et de la cavité et fonction de l'atténuation intrinsèque des formations sous-jacentes. Elle peut aussi se manifester par des discontinuités latérales des réflexions ou par l'apparition d'hyperboles en fonction de la forme et des dimensions de la cavité. Cette méthode est cependant fortement influencée par la conductivité de l'encaissant qui provoque l'atténuation du signal et limite la profondeur d'investigation [18].

Afin d’éliminer le bruit et les artéfacts qui interfèrent avec les signaux issus des cibles enfouies, des traitements appropriés ont été appliqués aux données brutes recueillies à l’aide du radar géologique. Ces traitements qui ont notamment pour but d'augmenter le rapport signal/bruit, incluaient entre autres : (a) une correction statique visant à localiser le temps zéro des enregistrements, (b) un filtrage fréquentiel passe-bande qui a pour objectif d'éliminer les hautes fréquences affectées par le bruit et, (c) un gain adéquat permettant de rehausser les signaux en amplifiant les réflexions masquées par l’atténuation ou par des effets latéraux.

Ces deux méthodes géophysiques sont très utilisées dans les reconnaissances des terrains de sub-surface. Elles ont toutes les deux l'avantage d'offrir une très bonne résolution de cartographie des hétérogénéités superficielles tout en étant non destructives. C’est ce qui explique d'ailleurs le choix de leur mise en œuvre dans la présente étude. Leur application à la détection des cavités souterraines en milieu urbain a donné des résultats très satisfaisants dans divers contextes d'utilisation [4, 7, 14]. On doit signaler, en particulier l'expérimentation 
conjointe très concluante de ces deux techniques, dans l’ancienne médina de Béni-Mellal qui se situe à une cinquantaine de kilomètres au Sud-Ouest de Zaouit Ech Cheikh et où se pose le même problème. En effet, des cavités mises en évidence par méthodes géophysiques dans cette médina ont été confirmées par sondage [16]. Toutefois, on doit reconnaître l'existence de certaines limites à ces techniques de reconnaissance indirectes. La plus handicapante pour les deux méthodes est la présence d’un recouvrement conducteur qui réduit considérablement leur profondeur de pénétration.

\section{Résultats et discussion}

Les résultats des mesures géophysiques enregistrées le long des profils E1 et E2 sont présentés en figure 3. Nous avons choisi ces deux profils car ce sont les seuls qui permettent de confronter les données des deux techniques mises en œuvre. Les mesures de tomographie électrique sont présentées sous forme de pseudosections ou panneaux dont l'inversion a permis d'établir des modèles de résistivité électrique du sous-sol (Fig. 3a,f). Ces modèles montrent d’importantes variations latérales de la résistivité électrique des terrains de fondations de la ville de Zaouit Ech Cheikh. Parmi ces variations, on s’intéresse en particulier aux zones très résistives représentées par les couleurs rouge à mauve de la palette utilisée. Cet intérêt est justifié par le fait que toutes les cavités reconnues dans la ville de Zaouit Ech Cheikh sont vides (càd remplies d’air) et devraient, par conséquent, engendrer des variations positives de résistivité. Les zones résistives mises en évidence présentent des formes, des tailles et des profondeurs variables. Leur dimension et la profondeur de leur centre sont généralement plus importantes au niveau du profil E1. Les données du radar géologique confirment l’hétérogénéité du sous-sol du site étudié qui se manifeste par une grande variation d'amplitude des échos enregistrés (Fig. 3b,g). En effet, sur l'ensemble des sections, on observe des zones de forte énergie qui alternent avec des plages plus calmes. 
Par ailleurs, afin de vérifier la signature radar de ces cavités éventuelles, les plages résistives ont été systématiquement délimitées, numérotées et superposées aux radargrammes (Fig. 3c,h). L’analyse conjointe des enregistrements radars et des anomalies de résistivité permettent de délimiter plusieurs cavités souterraines (Fig. 3e,j).

En complément de l'étude géophysique, nous avons mené une enquête en vue de rassembler le maximum d'informations sur l'existence de cavités souterraines dans la zone prospectée. Cette enquête consistait d'abord à recueillir des renseignements auprès des habitants qui avaient une connaissance de certaines cavités découvertes suite à des creusements de la voie publique pour divers travaux, tel que l'installation de canalisation ou de l’enterrement de câblages. Ensuite, il a fallu procéder à la cartographie détaillée des indices d’instabilités ou d'affaissements visibles sur certains bâtiments et sur les voiries. Les résultats de cette enquête pour les profils E1 et E2 sont présentés en cavités souterraines en figure 3d,i.

La concordance des données géophysiques et des informations issues de l'enquête conforte l'hypothèse de l'existence de cavités souterraines, comme c'est le cas pour les anomalies de résistivité 3, 4 et 5 et 9 et 10 respectivement sur les profils E1 et E2 que nous avons interprétées comme des cavités (Fig. 3e,j). Toutefois, les renseignements fournis par l'enquête n’ont été considérés qu'à titre indicatif vu l'imprécision sur la localisation des cavités. Ainsi, les zones résistives 2 sur le profil E1 et 5 sur le profil E2, qui coïncident avec des réflexions de forte amplitude, correspondraient à des vides souterrains bien qu'elles ne soient pas parfaitement centrées sur les cavités soulignées par l'enquête. En outre, compte tenu du fait que les résultats de l'enquête ne permettent pas de mettre en évidence toutes les cavités enfouies, nous interprétons en tant que cavités souterraines les anomalies 1 et 2 du profil E1 et 6 du profil E2 car elles correspondent à des zones résistives qui coöncident avec des réflexions de forte énergie (Fig. 3c,h). Signalons par ailleurs que la plus importante zone résistive qui correspond à l'anomalie 1 sur le profil E1, ne correspondrait pas à un vide 
souterrain car elle se superpose sur le radargramme à un litage parfaitement horizontal qui reflète vraisemblablement l'aspect sein des terrains de fondation de la ville de Zaouit Ech Cheikh.

Les cavités souterraines mises en évidence par la présente étude au niveau des profils E1 et E2 sont présentées en figure 3e,j. Ces cavités possèdent des formes différentes et de tailles variables. Certaines sont allongées verticalement, elle pourrait correspondre à des fosses septiques (C1, C’3 et C’4). Les autres cavités représenteraient soit des vides karstiques, soit des zones de prélèvements des matériaux gréseux de construction.

Signalons enfin que dans le cadre des travaux de recherche académiques que nous menons dans la région de Tadla-Azilal en général et dans la localité de Zaouit Ech Cheikh en particulier, nous ne disposons pas des moyens et des autorisations nécessaires pour valider les anomalies géophysiques mises en évidence. Le seul élément de validation disponible à présent c’est l’effondrement partiel, survenu récemment, du toit de la cavité C2 détectée sur le profil E1 (Photo 3). Toutefois, d'autres arguments peuvent être avancés pour appuyer les interprétations proposées :

- $\quad$ le recoupement des deux méthodes géophysiques utilisées ;

- l'existence d'indices d'instabilités de surface visibles au niveau des constructions et des infrastructures (voieries, canalisations, etc...);

- $\quad$ le contrôle par sondage d'anomalies similaires et dans un contexte identique au niveau de la ville de Béni-Mellal, a permis la mise en évidence de cavités souterraines [16].

\section{Conclusion}

Les résultats des premières expérimentations de la prospection géophysique entreprises dans la ville de Zaouit Ech Cheikh illustrent l'intérêt de la tomographie électrique et du radar géologique dans la détection et la délimitation des cavités souterraines. Elles montrent également que ces deux méthodes sont bien adaptées à la reconnaissance du sous-sol 
en milieu urbain. Les résultats très encourageants que nous avons obtenus, nous permettront d'étendre la couverture géophysique à l'ensemble du périmètre urbain de la ville de Zaouit Ech Cheikh dans l'objectif d'établir une carte détaillée des vides souterrains contenus dans ses terrains de fondation. L’élaboration d'un système d'information géographique qui inclurait, en plus de cette carte, l'ensemble des données se rapportant aux instabilités du sous-sol dans cette ville, permettra de prévenir les risques d’effondrements des constructions.

Par la suite, la reconnaissance géophysique pourra se généraliser à l’ensemble des superficies concernées par les futurs programmes d’aménagement autour de la ville de Zaouit Ech Cheikh. On pourra ainsi agir bien en amont et éviter les conséquences désastreuses des instabilités engendrées par les cavités souterraines.

\section{Remerciements}

Ce travail a été réalisé dans le cadre du projet OTAN (EST.CLG 980288) entre l’université Cadi Ayyad (Maroc) et l'université de Barcelone (Espagne). Nous remercions le personnel de la commune de Zaouit Ech Cheikh pour leur contribution à l'acquisition des données sur le terrain ainsi que les deux rapporteurs anonymes pour leurs remarques constructives. 


\section{Références}

[1] W. Al-Fares, M. Bakalowicz, Y. Alboury, J.-M. Vouillamoz, M. Dukhan, G. Toe, R. Guerin, Contribution de la géophysique à l'étude d’un aquifère karstique - Exemple: le site karstique du Lamalou, $3^{\text {ème }}$ Colloque GEOFCAN, Orléans (2001) 25-26 Septembre.

[2] P. Azemard., J.L. Garciaz, Recherche des karstifications sous la plate-forme du TGV Méditerrannée, Actes Journ. Scient. Et Techn. Radar, Nantes , France (2001), mai, 4p.

[3] J. Bourcart, Notice explicative de la carte géologique d'exploration du territoire autonome du Tadla au 1/200.000. Notes Mém. Serv. Géol. Maroc 58 bis (1942), 44p.

[4] A. Casas, R. Lazaro, M. Vilas, E. Busquet, Detecting karstic cavities with ground penetrating radar at different environments in Spain. Proceedings of $6^{\text {th }}$ International Conference on Ground Penetrating Radar, Japan (1996), 455-460.

[5] D. Clark, Near surface effects workshop. The leading Edge, 6 (1987), 8-9.

[6] G. Choubert, A. Faure Muret, Lexique stratigraphique du Maroc. Notes Mém. Serv. Géol. Maroc 134 (1956), 165p.

[7] S. Deng, Z. Zuo, H. Wang, The application of ground penetrating radar to the detection of shallow faults and cavities. Proceedings of $5^{\text {th }}$ International Conference on Ground Penetrating Radar, Ontario, Canada, 3 (1994), 1115-1120.

[8] J. Deceuster, O. Kaufmann, Application des tomographies en résistivité électrique 3D à la reconnaissance de zones karstifiées, Belgique, $4^{\text {ème }}$ Colloque GEOFCAN, Paris (2003), 23-24 Septembre.

[9] J.C. Dubois, Borehole radar experiment in limestone: analysis and data processing, First Break 13, 2 (1995), 57-67. 
[10] L.S. Edwards, A modified pseudosection for resistivity and induced polarization. Geophysics 42 (1977), 1020-1036.

[11] L. Falk, O. Olson, E. Sandberg, O. Forslund, L. Lundmark, A directional antenna for borehole radar. Proceedings of the $3^{\text {rd }}$ OECD/NEA Stripa Symposium, Stockholm (1989), 61-72.

[12] R. Lagabrielle, P. Grandsert, S. Millereau, S. Nebieridze, Performances comparées de méthodes géophysqiues pour la détection de cavités dans les limons. Exemple de la station d'essais de la SNCF sur la LGV Nord, Actes Journ. Scient. Et Techn. Radar, Nantes , France (2003), octobre, 4p.

[13] M.H. Loke, R.D. Barker, Rapid least-square inversion of apparent resistivity pseudosection by a quasi-Newton method. Geophysical Prospecting, 44 (1996), 131152.

[14] G. A. McMechan, R. G. Loucks, X. Zeng, P. Mescher, Ground penetrating radar imaging of a collapsed paleocave system in the Ellenburger dolomite, central Texas. Journal of Applied Geophysics, 39 (1998), 1-10.

[15] J.M. Miehe, B. Feuga, C. Vachette, Détection d'une cavité remplie de saumure par imagerie électrique, $4^{\text {ème }}$ Colloque GEOFCAN, Paris (2003), 23-24 Septembre.

[16] A. Najine, M. Jaffal, T. Aïfa, M. Filahi, A. Arioua, A. Boukdir, P. Andrieux et F. Regiba, Reconnaissance de cavités souterraines par tomographie électrique et radar géologique dans le centre ville de Béni-Mellal (Maroc). Bulletin des Laboratoires des Ponts et Chaussées, 260 (2006), 83-89.

[17] D.S. Parasnis, Principles of applied geophysics, Fifth Edition, Chapman \& Hall (Eds) London (1997), 429p. 
[18] G. Pottecher, A synthetic pulse GPR based on a network analyzer. Third International Conference on Ground Penetrating Radar, Denver, May (1990), 14-18.

[19] M.J.S. Roth, J.R. Mackey, C. Mackey, J.E. Nyquist, A case study of the reliability of multielectrode earth resistivity testing for geotechnical investigations in karst terrains, Engineering Geology 65 (2002), 225-232.

[20] W.M. Telford, L.P. Geldart, R.E. Sheriff, Applied Geophysics, Second Edition, Cambridge University Press (1995), 770p. 


\section{Légende des figures}

\section{Figure captions}

Figure 1. Localisation et géologie de la zone d'étude. 1. Permo-Trias : basaltes doléritiques, marnes et argiles à niveaux salifères ; 2. Lias inférieur-Domérien : dolomies et calcaires dolomitiques ; 3. Toarcien-Aalénien : dolomies ; 4. Aalénien-Dogger : calcaires ; 5. Crétacé : grès, marnes rouges et calcaires ; 6. Tertiaire et Quaternaire ; 7. Faille ; 8. Rivière.

Figure 1. Location and geology of the study area. 1. Permian-Trias: doleritic basalts, marls and clays with salty levels; 2. Lower Lias-Domerian: dolomites and dolomitic limestones; 3. Toarcian-Aalenian: dolomites; 4. Aalenian-Dogger: limestones; 5. Cretaceous: sandstones, red marls and limestones; 6. Tertiary and Quaternary; 7. Fault; 8. River.

Figure 2. Localisation des profils de mesures géophysiques sur un plan de la Ville de Zaouit Ech Cheikh montrant l'état des constructions. 1. Périmètre urbain, 2. Construction, 3. Construction présentant des indices d’instabilités, 4. Profil électrique, 5. Profil de radar géologique.

Figure 2. Location of the geophysical measurements profiles on a map of the Zaouit Ech Cheikh city showing the state of buildings. 1. Urban perimeter, 2. Buildings, 3. Buildings showing signs of instabilities, 4. Electrical profile, 5. GPR profile.

Figure 3. Résultats des mesures géophysiques enregistrées le long des profils E1 (a, b, c, d et e) et E2 (f, g, h, i et j). a,f : Tomographie électrique; b,g : Radar géologique ; c,h : superposition des anomalies de résistivité aux sections du radar géologique, d,i : Informations issues des enquêtes de terrain ; e,j : Interprétation.

Figure 3. Results of geophysical measurements recorded along profiles E1 (a, b, c, d and e) and E2 (f, g, h, i and j). a,f: Electrical tomography; b,g: GPR; c,h: superposition of resistivity anomalies to GPR sections; d,i: Information from field investigations; e,j: Interpretation. 
Photo 1. Effondrement engendré par une cavité souterraine.

Photo 1. Collapse caused by an underground cavity.

Photo 2. Levé du radar géologique à l’aide d’un équipement Ramac.

Photo 2. GPR data acquisition using a Ramac equipment.

Photo 3. Effondrement du toit de la cavité souterraine C2 mise en évidence au niveau du profil E1. L’emplacement de la photo est indiqué sur la figure 3e.

Photo 3. Collapse of the top of the underground cavity C2 detected on profile E1. The shot is located on figure 3e. 


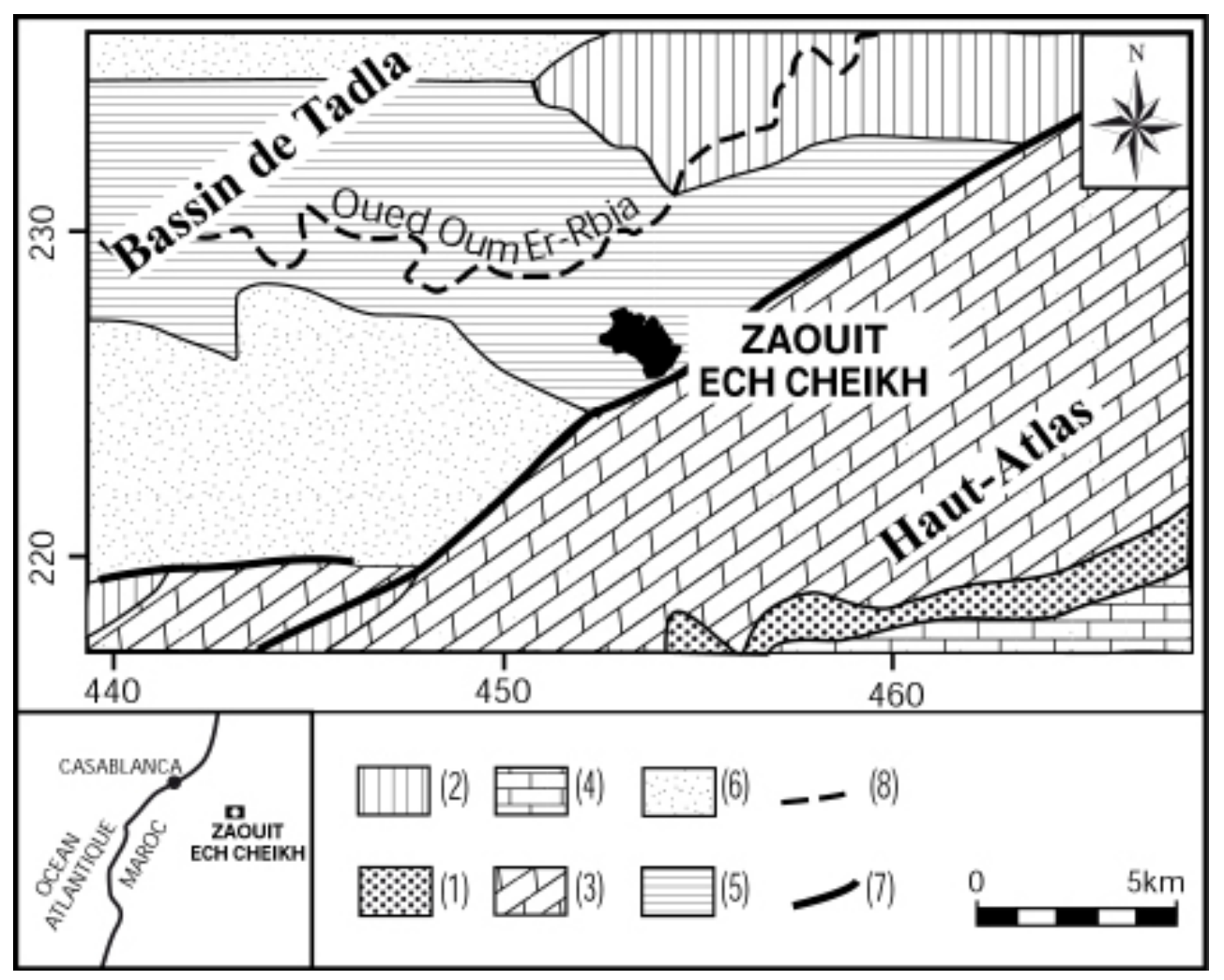

Figure 1

El Khammari et al. 


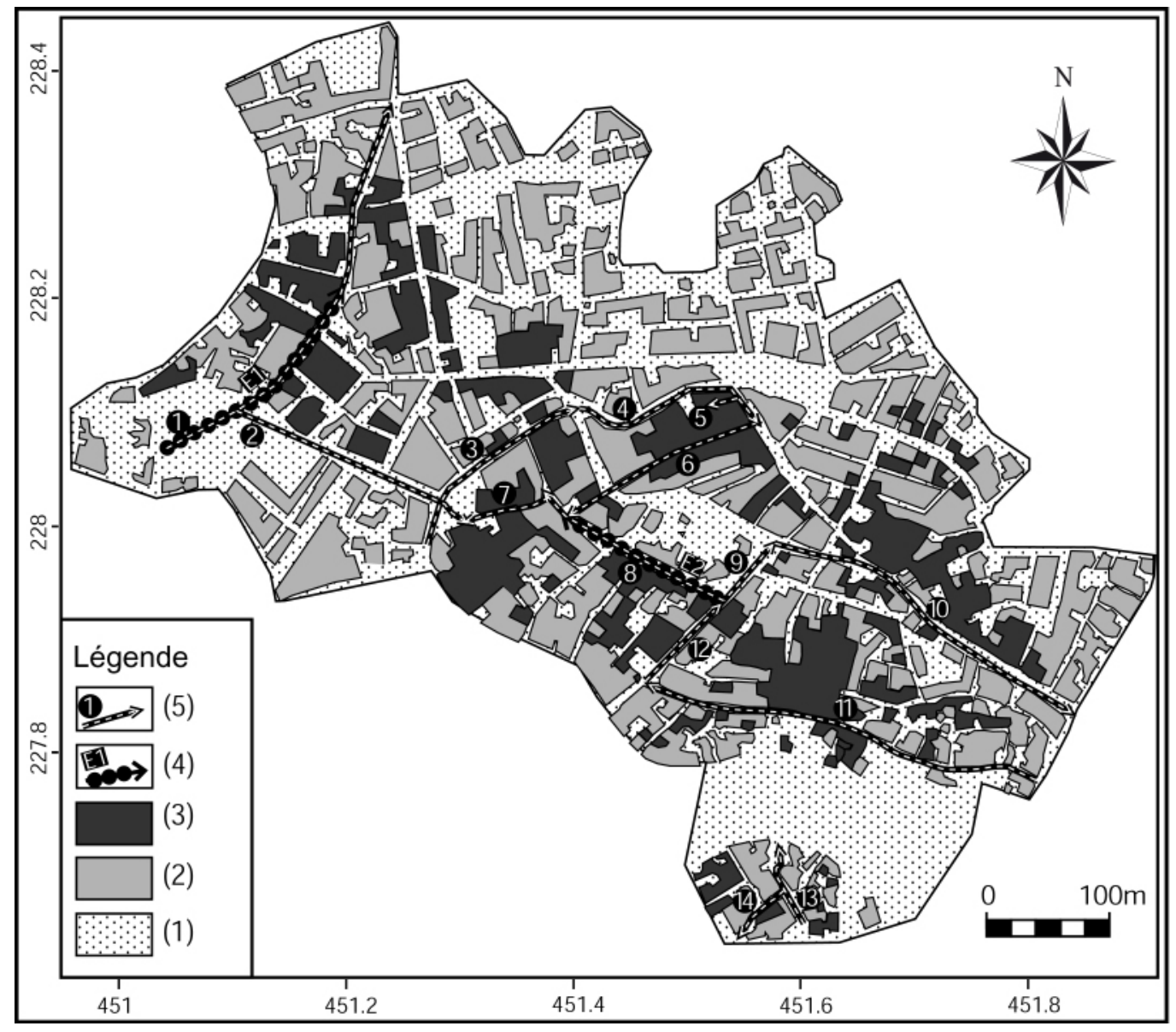

Figure 2

El Khammari et al. 

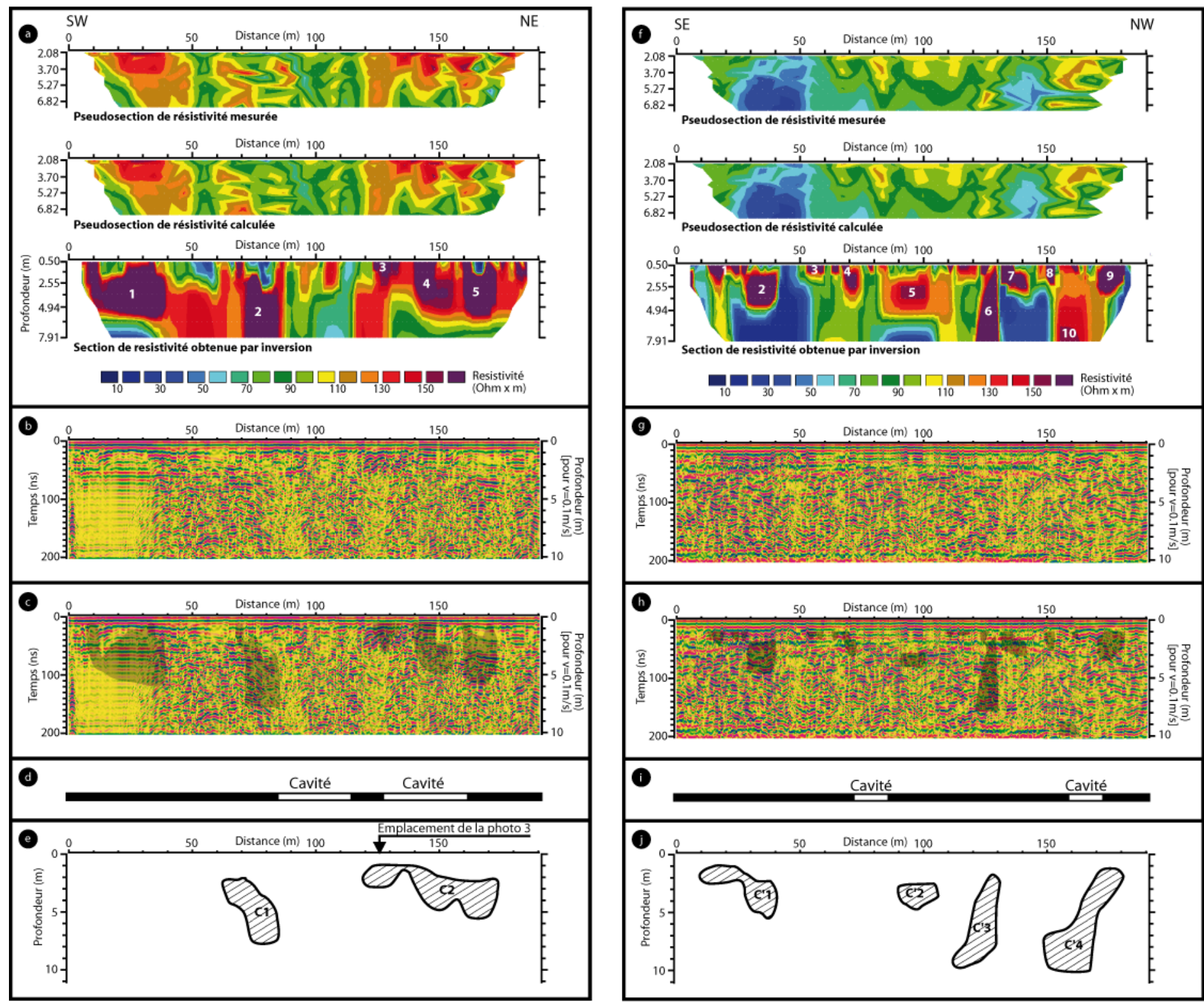

Figure 3

El Khammari et al. 


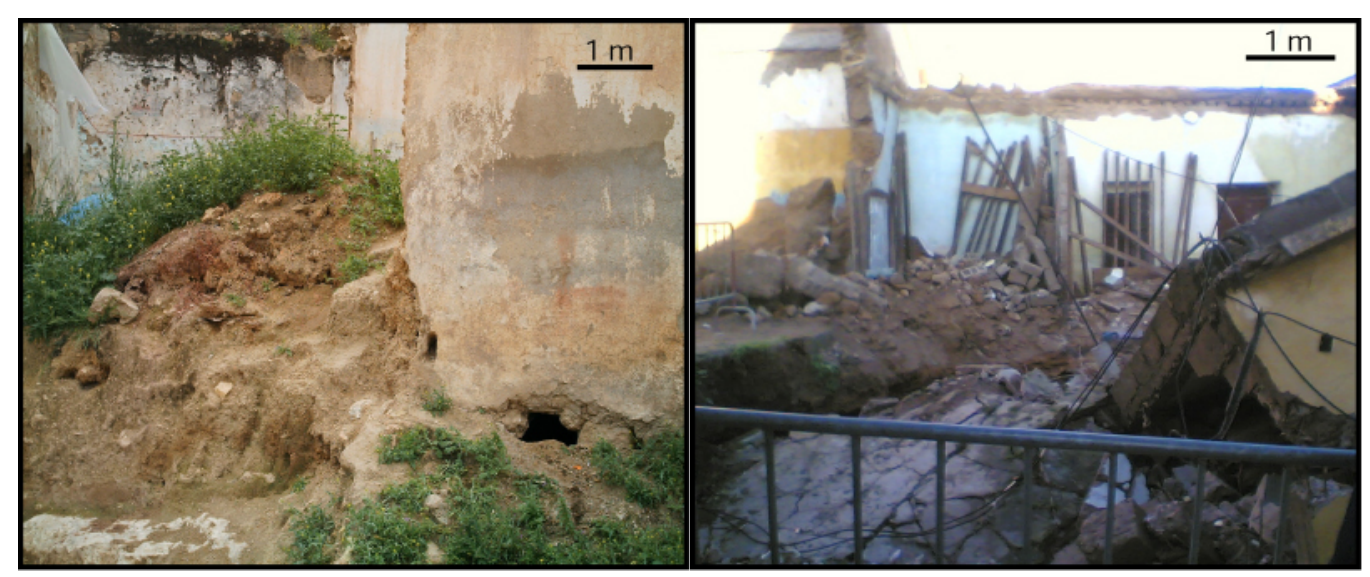

Photo 1

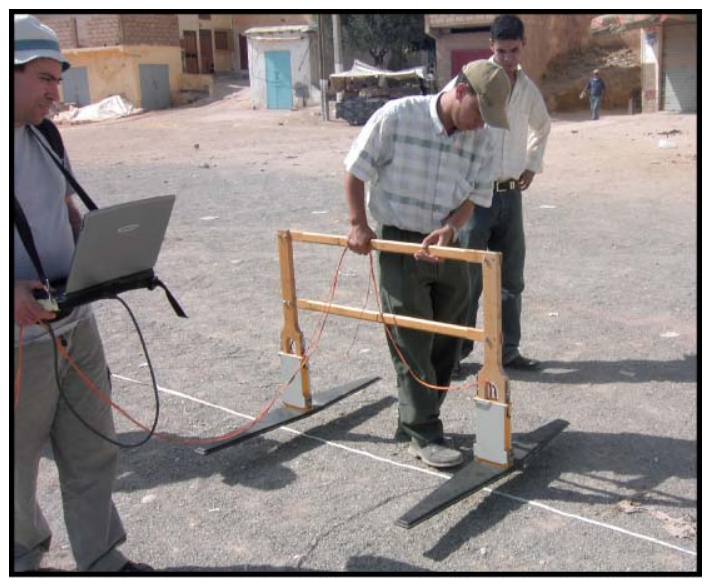

Photo 2

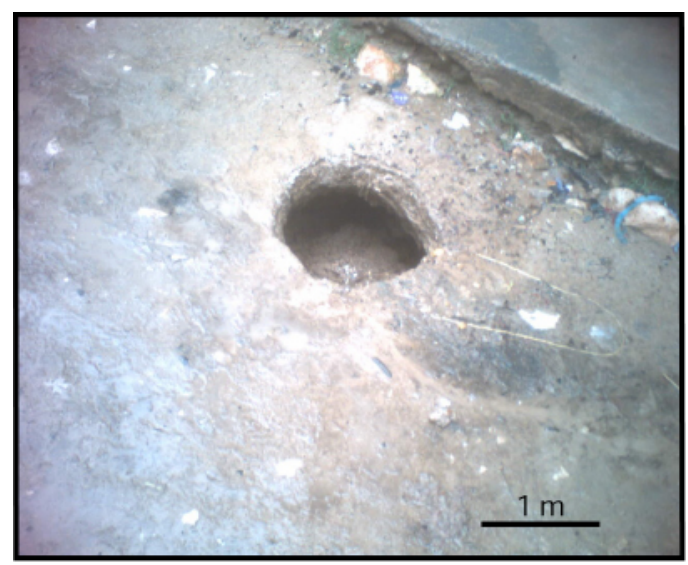

Photo 3

El khammari et al. 